\title{
DESARROLLO DEL APRENDIZAJE BASADO EN PROBLEMAS A TRAVÉS DE CASOS CLÍNICOS EN SEMIOLOGÍA PARA ESTUDIANTES DE ENFERMERIÁA
}

Recibido: agosto del 2018

Aceptado: abril del 2019

Singri Elena Rosado Ferreira ${ }^{1}$, Alexander Ortiz Ocaña ${ }^{2}$

\section{Resumen}

El presente artículo tiene como objetivo principal presentar los resultados de la implementación del aprendizaje basado en problemas (ABP) como estrategia didáctica de integración de los conocimientos teóricos mediante la práctica en la asignatura de Semiología en el programa de formación en Enfermería de la Universidad del Magdalena. La investigación se realizó con base en una metodología mixta e introducción de elementos de participación-acción y mediando casos clínicos para el desarrollo de las habilidades del estudiante en la valoración sistemática del paciente. Se realizó un trabajo de índole cuasiexperimental sobre una muestra de 21 estudiantes de cuarto y quinto semestre, basado en el diseño de cinco casos clínicos. Posteriormente y mediante la aplicación de una encuesta tipo Likert de escala 1 a 7 en conocimientos, habilidades y actitudes, con un total de 54 ítems, se determinaron el acuerdo, la aceptación y la tendencia actitudinal respecto al uso de dicha estrategia didáctica con base en ABP como alternativa de cambio. Los resultados permitieron evidenciar el acuerdo pleno de los estudiantes con los criterios esperados por el ABP, así como la comprobación de su plena disposición respecto a la metodología implementada. En general, el grupo evidenció comprender el aporte de la orientación diferencial que provee el uso del ABP mediante los casos clínicos propuestos en el aula para el desarrollo de la asignatura de Semiología.

Palabras clave: aprendizaje basado en problemas, casos clínicos, enfermería, semiología.

\footnotetext{
1 Enfermera, magíster en Educación, candidata a doctora en Ciencias de la Educación. Docente catedrática de la Universidad del Magdalena. Correo: chonirofe@hotmail.com

2 Doctor en Educación. Docente de tiempo completo de la Universidad del Magdalena. Correo: alexanderortiz2009@gmail.com
} 


\section{DEVELOPMENT OF PROBLEM-BASED LEARNING THROUGH CLINICAL CASES IN SEMIOLOGY FOR NURSERY STUDENTS}

Singri Elena Rosado Ferreira ${ }^{1}$, Alexander Ortiz Ocaña ${ }^{2}$

\section{Abstract}

Our main objective is to introduce the results of the implementation of the problem-based learning method (ABP in Spanish) as a didactic strategy to integrate theorical knowledge and practice in the course of Semiology in the Nursery program of Madgalena University. Research was based in a mixed methodology, with an introduction of elements of participative-action and through the management of clinical cases for the development of students' skills in the assessment of patients. We carried out a quasi-experimental work on a sample of 21 students of fourth and fifth semester, based on the design of five clinical cases. After that, we applied a Likert-based survey with a scale of 1 to 7 in knowledge, abilities, and attitudes, with a total of 54 items. We determined the agreement, acceptance and attitude trend regarding the use of the didactic strategy based in $\mathrm{ABP}$ as an alternative for change. Results allowed us to evidence how the students agreed with the expected criteria of ABP, as well as to prove their willingness to use the implemented methodology. In general, the group showed that they understood the contribution to differential orientation provided by the usage of ABP through the clinical cases proposed in the classroom to carry out the course of Semiology. 


\section{DESENVOLVIMENTO DA APRENDIZAGEM BASEADA EM PROBLEMAS A PARTIR DE CASOS CLÍNICOS EM SEMIOLOGIA PARA ESTUDANTES DE ENFERMAGEM}

Singri Elena Rosado Ferreira ${ }^{1}$, Alexander Ortiz Ocaña ${ }^{2}$

\section{Resumo}

Este artigo tem como objetivo principal apresentar os resultados da implantação da aprendizagem baseada em problemas (ABP) como estratégia didática de integração dos conhecimentos teóricos mediante a prática na disciplina de Semiologia, no programa de Enfermagem da Universidad del Magdalena, Colômbia. Esta pesquisa foi realizada com base em uma metodologia mista e em uma introdução de elementos de participação-ação e mediando casos clínicos para desenvolver habilidades do estudante na avaliação sistemática do paciente. Foi realizado um trabalho de índole quase-experimental com uma amostra de 21 estudantes de quarto e quinto período, baseado no desenho de cinco casos clínicos. Em seguida, mediante a aplicação de um questionário tipo Likert de escala de 1 a 7 em conhecimentos, habilidades e atitudes, com um total de 54 itens, foram determinados o acordo, a aceitação e a tendência atitudinal a respeito do uso dessa estratégia didática com base na $\mathrm{ABP}$, como alternativa de mudança. Os resultados permitiram evidenciar o acordo pleno dos estudantes com os critérios esperados pela $\mathrm{ABP}$, bem como a comprovação de sua plena disposição quanto à metodologia implantada. Em geral, o grupo evidenciou compreender a contribuição da orientação diferencial que fornece o uso da $\mathrm{ABP}$ mediante casos clínicos propostos na sala de aula para desenvolver a disciplina de Semiologia. 


\section{Introducción}

El método del ABP tiene su origen y sus primeras aplicaciones prácticas durante la década de los años sesenta, en la Escuela de Medicina en la Universidad de Case Western Reserve de los Estados Unidos, como también en la Universidad de McMaster en Canadá. El desarrollo de esta metodología se produjo con el objetivo específico de mejorar la calidad educativa en el campo médico, de acuerdo con el Instituto Tecnológico y de Estudios Superiores de Monterrey, ITESM (2000), al buscar reorientarla pasando del currículum basado en exposiciones magistrales de los temas a una didáctica más integrada a problemas de la vida real, al poner en juego diversas áreas del conocimiento en la búsqueda de soluciones a cada problema planteado.

En Colombia, los procesos educativos utilizados han soportado numerosos cambios con miras a su mejoramiento, a través de lo que se suponen reformas educativas como políticas públicas indicativas. En la educación superior estas reformas buscan trabajar por una calidad y excelencia académica. Según el Ministerio de Educación (2016), Colombia actualmente enfrenta dos retos cruciales: cerrar las brechas existentes en términos de participación y mejorar la calidad de la educación para todos.

Dentro de tal empeño, la Universidad del Magdalena no es ajena a ese mejoramiento continuo, al estar siempre abierta a la adquisición y apropiación de procesos educativos que contribuyan al ase-

guramiento de las calidades didácticas y pedagógicas en general. En este sentido, el presente trabajo estuvo orientado hacia uno de los modelos implementados con éxito a nivel de programas de formación en ciencias de la salud, reconocido como aprendizaje basado en problemas (Project Based Learning, BPL, por sus siglas en inglés). Su éxito radica a modo de enfoque metodológico en que, a diferencia de la enseñanza tradicional, no depende del aprendizaje memorístico e intenta resolver la necesaria conexión entre el conocimiento adquirido y su adaptación e integración con otros saberes de cada disciplina. El ABP es considerado un enfoque innovador, ya que establece sus raíces en el constructivismo, de manera que el estudiante a partir de un problema o del planteamiento de una situación problemática elabora el conocimiento, como lo han expresado Fernández, García, De Caso, Fidalgo y Arias (2006).

De acuerdo con lo anterior, el resolver problemas en situaciones concretas establecidas es la meta final del ABP como metodología de enseñanza y aprendizaje. La eficacia del ABP ha quedado demostrada en la enseñanza superior impartida en universidades de todo el mundo, como en el caso del proceso de transformación y convergencia conocido como Espacio Europeo de Educación Superior (Martin, Martin y Cobo, 2012).

En comparación con el aprendizaje tradicional, el ABP parece ser más efectivo y estimulante, sin embargo, es necesario continuar investigando y mejorando, ya que hasta ahora ha sido aplicado de manera parcial en la educación superior dentro del ámbito de formación de profesionales de la salud (medicina, enfer- 
mería, obstetricia, entre otros), en tanto que, en otras áreas y etapas educativas, su aplicación está menos extendida (Fernández, et al., 2006).

La investigación partió de formularse el problema acerca de si la implementación de una estrategia didáctica basada en ABP, específicamente en el campo de la semiología dentro de un programa de enfermería, representaría una alternativa de integración de los conocimientos teóricos con la práctica en el proceso de aprendizaje de los estudiantes. El presente artículo, basado en un proyecto doctoral previo, expone los resultados del objetivo de implementar el $\mathrm{ABP}$ en la asignatura de Semiología del programa de Enfermería de la Universidad del Magdalena, en búsqueda de la integración de los conocimientos teóricos con la práctica de los estudiantes como una alternativa para generar cambios y mejoramientos en el nivel de aprendizaje de dichos estudiantes.

En el planteamiento del problema se coincide con lo que ha planteado Olivares (2010) de la Universidad de Nayarit, acerca de que el modelo tradicional ha resultado ineficiente para seguir el ritmo que marcan las necesidades de la sociedad contemporánea, debido a que este es sinónimo de una espera para que alguien transmita el conocimiento, a manera de simple transferencia y que, de alguna manera, ya procesado o digerido por alguien como el docente, este a su vez deba lograr el alcance del estímulo necesario para que el estudiante pueda y desee adquirir dicho conocimiento.

Mediante la introducción del ABP, como construcción didáctica y meto- dológica, dentro de la enseñanza de la semiología se esperaba generar un mayor nivel de aprendizaje efectivo en los estudiantes, a través de sus propias experiencias y de la positiva acción de compartirlas, tanto con sus compañeros como con el mismo profesor. La inserción exitosa de un proceso educativo relativamente nuevo y creativo para la universidad y el programa, conlleva que el estudiante se empodere de su propio aprendizaje, al lograr afianzar conductas y habilidades de autoaprendizaje, autocrítica, inventiva y capacidad para trabajar grupalmente en la construcción de aquellos nuevos saberes que le permitirán adquirir las competencias esperadas al final del curso correspondiente.

La investigación desarrollada lo consideró una metodología mixta. Fue cuantitativa desde la perspectiva cuasi experimental y cualitativa desde la inclusión de elementos de participación-acción, con un enfoque científico interpretativo-comprensivo que permitió el análisis del proceso de implementación del $\mathrm{ABP}$ en los estudiantes de Enfermería.

La perspectiva acometida se acoge a lo que Castrillón (2002) ha manifestado en el sentido en que la interacción entre profesión y vocación en enfermería ha sido concluyente. En este sentido, la mayor parte del siglo XX dominó la vocación expresada en la profesión, pero esta fusión ha estado experimentando cambios en las dos últimas décadas y el futuro será muy diferente del presente. Esa diferencia estará marcada por un desarrollo de la disciplina, en el cual la investigación y la sistematización de las 
prácticas profesionales ocuparán un papel fundamental.

\section{Metodología}

En el proceso investigativo planteado se asumió una concepción tríadica e integradora desde lo epistemológico: aprendizaje basado en problemas (Branda, 2004), enfermería (Acevedo, 2009) y didáctica, a partir de un análisis ontológico, epistémico, heurístico y teleológico del objeto de estudio. El trabajar con una metodología mixta y combinar paradigmas cuantitativos y cualitativos en lo cuasiexperimental, implica un trabajo de observación del comportamiento de los estudiantes como sujetos en ejercicio de su autonomía de aprendizaje, por parte del docente inmerso en un ámbito de investigación-acción, a partir de su intervención coordinadora con un enfoque científico, interpretativo-comprensivo y analítico de la incidencia del ABP en las características comportamentales de los estudiantes de Enfermería durante el desarrollo de la práctica de la asignatura.

La muestra estuvo conformada por los estudiantes de cuarto (11 alumnos) y quinto (10 alumnos) semestre del programa de Enfermería, para un total de 21 sujetos de muestra. En términos de instrumentos metodológicos fueron utilizados: una encuesta tipo Likert que contó con dieciséis (16) ítems en la categoría de conocimientos, diecinueve (19) ítems en la categoría de habilidades y diecinueve (19) ítems en la categoría de actitudes, cuya escala de valoración au-

214 mentaba de 1 a 7 , entre ningún acuerdo o total acuerdo, con la premisa corres- pondiente propuesta a los sujetos. La validez del instrumento se analizó previamente con base en consulta a expertos; por otra parte, se usó un formato para planteamiento y diseño de casos clínicos (anexos desde el A hasta el F).

Las técnicas utilizadas para el análisis interpretativo-comprensivo de los datos obtenidos fueron de estadística analítica y descriptiva, mediante frecuencias y medidas de tendencia central, para determinar curvas de tendencia radial y curvas de respuesta. El análisis cualitativo hizo contraste con los elementos derivados de revisión de la literatura acerca del tema teórico y conceptual subyacente.

\section{Resultados}

En general, los resultados demostraron que la metodología de casos fue de gran aceptación entre los estudiantes del grupo de muestra, para quienes significó una nueva e interesante experiencia. Fue una característica evidente durante el proceso en que los estudiantes del grupo acudieran a sus propios conocimientos, siendo compartidos en voz alta entre ellos mismos. Esto ha de significar metodológicamente que se produjo la utilización de todas sus habilidades individuales en pro del éxito del grupo, pero también la indudable actitud positiva por parte de cada uno para la identificación de técnicas exploratorias y concluir en la definición de mejores y correctos diagnósticos.

Para el desarrollo de las sesiones se propusieron cinco (5) diferentes casos clínicos: caso clínico 1: tórax, pulmón y piel 
(anexo a); caso clínico 2: cardiaco (anexo b); caso clínico 3: neurológico (anexo c); caso clínico 4: cabeza y cuello (anexo d) y caso clínico 5: abdomen (anexo e).

Antes de comenzar el proceso pedagógico con el grupo de estudiantes, se tuvieron en cuenta, de acuerdo con algunas de las precisiones metodológicas de la Open Course Ware (2012) de la Universidad de Murcia, los siguientes criterios de evaluación para cada participante:

1) Preparación para la sesión: utiliza fundamentalmente material relevante durante la sesión, se aplican los conocimientos previos, demuestra iniciativa personal, curiosidad y organización en su participación. Muestra evidencia de su preparación profesional en las sesiones de trabajo en grupo.

2) Participación y contribuciones al trabajo del grupo: su participación es constructiva y apoya al proceso del grupo. Demuestra su capacidad de dar y aceptar retroalimentación constructiva y contribuye a estimular el trabajo colaborativo.

3) Habilidades interpersonales y comportamiento profesional: demuestra habilidad para comunicarse con sus compañeros, escucha y atiende las diferentes aportaciones, es respetuoso y ordenado en su participación, además de colaborativo $y$ responsable.

4) Contribuciones al proceso de grupo: apoya el trabajo del grupo colaborando con sus compañeros y aportando ideas e información necesaria. Estimula la participación de sus compañeros o compañeras.

5) Actitudes y habilidades humanas: la persona es consciente de sus fuerzas y limitaciones, escucha las opiniones de los demás compañeros y compañeras, tolera los defectos de los demás y estimula el desarrollo de todos y cada uno de ellos.

6) Evaluación crítica: clarifica, define y analiza el caso problema propuesto, es capaz de generar y probar una hipótesis, identificando los objetivos de aprendizaje (OCW, 2012, p. 19).

De cada una de las sesiones correspondientes al análisis de los cinco casos clínicos, se tomaron evidencias en un total de 15 archivos videográficos. Los principales resultados de dicho trabajo de observación a las sesiones de análisis de casos fueron los siguientes:

- Se generó una plena disposición de los 21 estudiantes hacia la práctica de la metodología propuesta. En general, el grupo evidenció la plena comprensión de la orientación diferencial que provee el uso del $\mathrm{ABP}$ en el aula para el desarrollo de la asignatura de semiología.

- Los conocimientos de los estudiantes mostraron estar acordes a cada uno de los problemas planteados en los casos propuestos.

- Se percibieron inquietudes propias de toda novedad en un aula. Se tra- 
taba de una primera experiencia y el grupo mostraba signos de incertidumbre acerca del cómo reaccionar, de qué manera responder, acerca de cómo serían los turnos en la palabra, entre otras inquietudes de la coyuntura didáctica.

- Se contó con un ambiente pleno de respeto entre compañeros.

- No se presentó viso alguno de falsa competencia en cuanto a la pretensión de tener o no las respuestas o criterios más acertados.

- Cada una de las soluciones surgió de manera serena y con el criterio básico de llegar a una mejor opinión con carácter grupal.

- La discusión grupal fue calmada y centrada en la aportación de criterios orientados al objetivo diagnóstico.

- Los estudiantes aportaban hipótesis sin temor alguno a una posible refutación. Por el contrario, una eventual corrección u aporte era lo que cada uno esperaba de sus compañeros.

- En ningún momento se presentaron refutaciones dirigidas a las personas, estas solo se orientaron hacia las argumentaciones puestas en debate. En el sentido ya comentado, no se presentaron argumentaciones del tipo falacia de negación, de veracidad o de generalización, entre otras 216 que quisieran restar veracidad a una afirmación objetiva.
- Todos los estudiantes estuvieron siempre dispuestos a la escucha activa a la que hacen referencia Branda y Lee (2000).

La disposición tutorial se centró estrictamente en orientar a los estudiantes a conseguir su propia respuesta a cada caso propuesto. Respecto a esto, la tutoría docente buscó mantener una actitud neutra y de coordinación de las diversas ideas durante todo el proceso. Dicho rol tutorial fue de coordinación y orientación metodológica, mas no dirigida a la resolución del problema planteado en cada caso. Se alcanzó una gran voluntad de interacción entre estudiantes y docente durante la resolución de los casos expuestos.

\section{Discusión}

De acuerdo con Zhang (2014), el avance rápido de la tecnología médica y la ciencia requieren que la educación en enfermería se convierta en "híbrida" (p. 2), al combinar métodos tradicionales de enseñanza con metodologías innovadoras. La educación en enfermería ha comenzado a pasar del aprendizaje centrado en el docente al aprendizaje centrado en el alumno, aunque ambos siguen siendo quienes deben buscar activamente el conocimiento. El ABP ofrece una perspectiva más holística del problema en el propio entorno contextual de la enfermería y encaja bien con los conceptos del metaparadigma de persona, entorno, salud y enfermero (a), lo cual se evidenció claramente en el hecho de que los participantes objeto de estudio, durante la implementación del ABP con casos clínicos, mostraran plena satisfac- 
ción con lo obtenido como experiencia de aprendizaje.

Respecto al trabajo de observación realizado y con relación a las actitudes individuales, al considerar que se trató de una primera experiencia de la aplicación del ABP con casos clínicos para el grupo objetivo, se pueden resumir los siguientes resultados:

- Cada estudiante, hombre o mujer, mostró plena disposición a utilizar libremente su mirada y a tener contacto visual directo con sus compañeros o compañeras, así como con la docente.

- El lenguaje gestual fue de carácter espontáneo. Los gestos y las acciones con las manos fueron estrictamente los necesarios y siempre estuvieron orientados a realizar notas escritas o hacia el hacer las palpaciones necesarias al modelo didáctico "de torso" que fue utilizado en clase.

- Los estudiantes utilizaron tonos naturales en el manejo de su voz para entablar diálogos entre ellos. Se generó un clima de gran respeto al interior del grupo.

El ABP ha tenido una gran acogida y ha logrado expandirse en muchas instituciones universitarias del mundo (Martin, Martin y Cobo, 2012). En la Universidad del Magdalena este fenómeno no resultó ajeno y al implementar el PBL en el aprendizaje de la asignatura de semiología y a través de casos clínicos propuestos a los estudiantes de enfermería, se generó el debido interés.
Estos casos clínicos planteados como problemas estimularon el autoaprendizaje y la adquisición de conocimientos y habilidades que no hubieren sido fácilmente adquiribles en las clases puramente magistrales, según criterio hallado en los mismos estudiantes. Todos los estudiantes mostraron seguridad personal acerca de poder aprobar la asignatura mediante $\mathrm{ABP}$ y sin la tradicional metodología de clases magistrales. Al respecto y sin embargo, algunos autores como Fernández y Flores (2013), que han hecho parte de los grupos de investigadores interesados en la eficacia de aplicación pedagógica de la $\mathrm{ABP}$, con base en un estudio realizado en tutores y estudiantes en educación superior, han obtenido como resultados que algunos demuestran algún sesgo negativo mientras otros declaran una respuesta positiva frente a la eficacia de la estrategia ABP. De igual forma, Koutrouba y Karageorgou (2013), en un estudio sobre los resultados cognitivos y socio-afectivos del $A B P$, hallaron que los participantes consideraban que el $\mathrm{ABP}$ ofrecía una segunda oportunidad para desarrollar diversas habilidades cognitivas, considerables como cualidades profesionales y que las habilidades socio-afectivas se habían desarrollado en ellos durante la aplicación del ABP.

De acuerdo con lo mencionado, en este estudio se puede afirmar que desaparecieron los posibles prejuicios acerca de las competencias presumibles en cada uno de los compañeros de clase. Se observó un clima espontáneo de natural predisposición a los nuevos conocimientos. Persistió igualmente la expectativa de descubrimiento de lo desco- 
nocido, así como el reconocimiento del valor del criterio de los otros. La duración de los intercambios individuales de conocimientos de los estudiantes entre sí o de la exposición de sus criterios personales, respecto a cada uno de los casos que fueron planteados como material de estudio, discusión y diagnóstico, fue estrictamente la necesaria. La sensación general fue la de reconocer que los aportes de cada compañero eran igualmente valiosos y que sin ellos el resultado del diagnóstico esperado no hubiera sido el mejor.

No fueron introducidos en ningún momento, por parte de los estudiantes, temas ajenos a cada caso clínico en estudio, lo que demostró una plena concentración y atención al objeto de estudio. Respecto a ello, según Branda (2004), debe considerarse que hay una necesidad sentida en cuanto a diseñar guías que reflejen claramente las funciones del tutor docente, de los objetivos de aprendizaje y de las temáticas propuestas en cada uno de los casos o problemas utilizados como objeto de estudio en clase.

Con base en el recurso representado por las guías para los tutores, se puede pensar en dinamizar la tarea de estos docentes hacia la orientación y el enfoque pedagógicos que se propone para ABP. En el caso presente, los formatos de casos clínicos obraron de guía tutorial para el docente. La acción del tutor define la interacción entre los miembros del grupo y la relación práctica con sus conocimientos previos. No en

218 vano las raíces de un aprendizaje con base en el planeamiento de problemas están en el constructivismo, lo cual significa que el conocimiento previo del estudiante es base fundamental para su desempeño hacia la diagnosis del problema y su solución, pero a su vez, el estudiante, a partir del problema que se le propone y del planteamiento de una situación fundamentada en la diagnosis correcta, podrá elaborar el conocimiento posterior originado en la praxis.

\section{Conclusiones}

Los hallazgos de la investigación permitieron las siguientes conclusiones:

- Los casos clínicos en semiología para desarrollo del ABP, que favorecieron el desarrollo de las habilidades del estudiante para la valoración sistemática del paciente en el programa de formación en Enfermería de la Universidad del Magdalena, fueron: caso clínico 1: tórax, pulmón y piel; caso clínico 2: cardiaco; caso clínico 3: neurológico; caso clínico 4: cabeza y cuello, y caso clínico 5: abdomen. Estos llevan a los estudiantes a desarrollar estrategias que los estimulen a mejorar los procesos de autoaprendizaje y de pensamiento crítico.

- La mayoría de los estudiantes de cuarto y quinto semestre de Enfermería mostraron plena disposición a la implementación libre del PBL, a través de dichos casos en la asignatura de semiología.

- Aquellos estudiantes que al inicio de la implementación no mostraban 
aceptación por el PBL a través de casos clínicos, adquirieron expectativas positivas hacia el descubrimiento de lo desconocido, así como respeto a la importancia y el valor del criterio de los otros.

- Los casos clínicos planteados como problemas estimularon el autoaprendizaje y la adquisición de conocimientos y habilidades no fá- cilmente adquiribles en las clases magistrales.

El introducir el ABP como estrategia didáctica dentro del campo de la semiología en el programa de enfermería, significa una búsqueda de integración y de generar niveles de aprendizaje eficaz y científico en los estudiantes, organizando nuevos aspectos pedagógicos $\mathrm{y}$ didácticos. 


\section{Referencias bibliográficas}

Acevedo-Gamboa, F. (2009). Construcción metodológica para la enseñanza de la disciplina de enfermería: aspectos históricos y reflexiones. Investigación en enfermería: Imagen y DesarroIlo, 11(1), pp. 53-66. Recuperado de http://revistas.javeriana.edu. co/index.php/imagenydesarrollo/ article/view/1611/1031

Branda, L. (2004). El aprendizaje basado en problemas: Una herramienta para toda la vida. Madrid, España: Agencia Laín Entralgo.

Branda, L. y Lee, Y. (2000). Evaluación de la Competencia del Tutor. Bahía Blanca, Argentina: Universidad Nacional del Sur.

Castrillón, M. (2002). Desarrollo disciplinar de la enfermería. Revista Investigación y Educación en Enfermería, 20(1), pp. 82-88. Recuperado de: http://www.redalyc.org/ pdf/1052/105217873005.pdf

Fernández, S. \& Flores, M. (2013). Tutors' and Students' Views of Tutoring: A Study in Higher Education. In Back to the Future: Legacies, Continuities and Changes in Educational Policy, Practice and Research. M. A. Flores, A. A.Carvalho, F. I. Ferreira, and M. T. Vilaça, (eds), pp. 277-295. Rotterdam: Sense Publishers.

Fernández, M., García, S., De Caso, A., Fidalgo, R. y Arias, G. (2006). El aprendizaje basado en problemas: Revisión de estudios empíricos internacionales. Revista de Educación, 341, pp. 397-418. Recuperado de http://www.revistaeducacion.educacion.es/re341/ re341_17.pdf

Instituto Tecnológico y de Estudios Superiores de Monterrey, ITESM. (2000). El Aprendizaje Basado en Problemas como técnica didáctica. Monterrey, México: Dirección de Investigación y Desarrollo Educativo, Vicerrectoría Académica.

Koutrouba, K. \& Karageorgou, E. (2013). Cognitive and socio-affective outcomes of project-based learning: Perceptions of Greek Second Chance School students. Improving Schools, 16, pp. 244-260.

Martin, N., Martin, A. y Cobo, A. (2012). El aprendizaje basado en problemas (ABP) en gran grupo: una experiencia satisfactoria para los estudiantes de primero de grado de enfermería de Toledo. Docencia e Investigación, 37(22), pp. 127-38.

Ministerio de Educación. (2016). La educación en Colombia: revisión de políticas nacionales de educación. Recuperado de http://www. mineducacion.gov.co/1759/articles-356787_recurso_1.pdf

OCW. (2012). El proceso de evaluación en la metodología de Aprendizaje Basado en Problemas. Recuperado de http:// ocw.um.es/cc.-sociales/la-metodologia-de-aprendizaje-basado-en-problemas/material-de-clase-1/tema-6.pdf

Olivares, S. (2010). El aprendizaje basado en problemas. Una propuesta metodológica para transformar la universidad. Nayarit, México: Universidad Autónoma De Nayarit. Recuperado de http://www. congresoretosyexpectativas.udg. mx/Congreso $\% 202 /$ Mesa $\% 20$ $1 /$ d $\% 29 \% 20$ Aprendizaje $\% 20$ asistido\%20profesional/1.d.4.pdf

Zhang, W. (2014). Problem Based Learning in Nursing Education. Advances in Nursing, 2014, pp. 1-5. 


\section{Anexos}

\section{Anexo A. Caso clínico 1: piel, tórax y pulmón}

Paciente de sexo masculino de 56 años residente en lo alto de las Colinas del Pando (cerro), no hay control de vectores (insectos), queman basuras y queman cartón de empaques de huevo, todo para alejar los mosquitos.

Paciente fumador desde hace 20 años y de oficio reciclador.

\section{$\underline{\text { Antecedentes }}$}

EPOC, infarto agudo de miocardio, hipertensión arterial, mal estado bucal.

\section{$\underline{\text { Examen físico }}$}

En prácticas de salud familiar el paciente es identificado y valorado en ese instante y manifestaba: tos productiva de coloración verdosa, dificultad para respirar, fiebre elevada no cuantificada, diaforesis, dolor punzante de pecho, dolor muscular, diarrea de tres días, deshidratación muy marcada, cianosis central.

A la auscultación se perciben ruidos agregados: sibilancias, las cuales predominan al final de la inspiración, presenta tos, vibraciones fisiológicas y estertores crepitantes.

Signos vitales: respiración $28 \mathrm{x}^{\prime}$, frecuencia cardiaca $125 \mathrm{x}^{\prime}$, presión arterial sistólica menor $70 \mathrm{mmhg}$.

\section{Neumonía adquirida en la comunidad}

Se comporta con un $\mathrm{PH}$ menor 7,35, cuadro hemático menor del $30 \%$, urea aumentada de 20 muestra leucosistosis, soplo bronquial, lleva más de cuatro días de evolución, requiere hospitalización y técnicas explorativas, recuerda la topografía del tórax y se valora la piel.

\section{Definición}

Enfermedad infecciosa que ataca a los pulmones, especialmente a los individuos que no hayan sido recientemente hospitalizados; afecta a todas las edades, por lo general al inhalar patógenos fuera del ambiente hospitalarios.

Manifestaciones clínicas: fiebre, tos, fatiga, dificultad para respirar y dolor en el pecho. Los laboratorios son confirmativos en la hospitalización.

\section{Anexo B. Caso clínico 2: cardiaco}

Paciente de sexo femenino de 75 años se observa muy adinámica, con demencia senil, hipertensión arterial con enfermedad de base: diabetes mellitus tipo II, exfumadora, con dolor torácico de cuatro a seis meses, sudoración intensa, ligera disnea, dolor iniciado dos horas antes, tuvo desmayos, vértigo, con actividad.

Signos vitales: tensión arterial 140/80 $\mathrm{mmHg}$, frecuencia cardiaca $80 \mathrm{x}^{\prime}$. 


\section{Antecedentes}

No alergias, tratamiento con Amlodipino, dislipemia en tratamiento con Simvastatin.

Ortopnea positiva (problemas respiratorios al hacer ejercicios).

\section{Exámen físico}

Palidez mucocutánea, tórax hiperinsuflado, silbido hacia las arterias carótidas, cuello corto no bocio y tos con sangre.

\section{Estado actual}

Arrítmico con soplo sistólico en borde esternal. Ruido irradia al cuello, ausencia del segundo ruido y palpitaciones.

Abdomen globoso sin megalias, masa pulsátil en zona periumbilical.

Extremidades sin edemas, pulso difícilmente palpable, pulso capilar positivo, signo de martillo de agua positivo.

Insuficiencia cardiaca, ruido de dos soplos, estenosis aórtica severa sintomatológica.

Tratamiento

Cirugía de recambio valvular aórtico, cateterismo cardiaco, técnicas explorativas: calidad del pulso del cuello.

\section{$\underline{\text { Medios diagnósticos }}$}

222 Electrocardiograma, prueba de esfuerzo y resonancia magnética.

\section{Anexo C. Caso clínico 3: neurológico}

Paciente de 77 años de sexo masculino que asiste a su consulta por temblor en sus manos, sobretodo en la mano derecha desde hace tres meses, lo presenta de forma constante en ambas manos de predominio el lado derecho; presenta inexpresividad facial, dificultad al ponerse de pie y lentitud al caminar.

En sus miembros, a la movilización muestra un aumento del tono en las piernas, rigidez en rueda dentada en los brazos, tiene parálisis de la mirada vertical.

Tiene un mes de estar comportándose muy ansioso, llora muy fácil, siente que lo están abandonando. Tiene tres días de insomnio, tenía un día de cambio del ritmo vigilia-sueño; últimamente su hermana menor lo visita, le saluda diciéndole "Matilde" y en la conversación que cursa entre el silencio, responde cuando le preguntan, muestra la mano repetidas veces porque se le mueve mucho, mientras su hermana cree que le muestra el anillo de matrimonio y esta se lo quita y se lo limpia pero no se percata del temblor fino, así muestra varias veces la mano y ella dice que se la pasa mucho tiempo sentado.

\section{$\underline{\text { Antecedentes familiares }}$}

Hipertensión, catarata

\section{Examen físico}

Presión arterial 80/70mmHg, pulso $75 \mathrm{x}^{\prime}$, frecuencia respiratoria $18 \mathrm{x}^{\prime}$, temperatura de $36,7^{\circ} \mathrm{C}$, no hubo más hallazgos clínicos. 


\section{Anexo D. Caso clínico 4: cabeza y cuello}

Paciente mujer de 35 años, mestiza, casada, de religión católica, estudios de secundaria, ocupación ama de casa, nació en Palenque.

\section{Motivo de consulta}

Agrandamiento de las manos, pies y rasgos faciales. Aproximadamente hace dos meses y medio viene notando el aumento del volumen de sus manos, pies y rasgos faciales; incremento del peso; disminución del apetito sexual; separación interdentaria; debilidad muscular; cefalea, tipo apresivo región fronto-temporal; actualmente 7/10 escala de EVA y disminución gradual de la visión.

\section{$\underline{\text { Antecedentes familiares }}$}

Diabetes mellitus

Examen fisico

Piel y faneras secas, gruesa con distribución del pelo

Órganos de los sentidos: disminución de la visión

Cardio: no registra

Respiratorio: apnea del sueño

Gastrointestinal: constipación

Genitourinario: no registra

Ano perineal: no registra

Endocrino: no registra
Hemolinfopoyético: no registra

Osteomuscular: debilidad muscular

Neurológico: hemianopsia bilateral

Hipertensión diagnosticada hace dos años, tratada con Losartan

Signos vitales: tensión arterial $125 / 80 \mathrm{mmHg}$, frecuencia cardiaca $75 \mathrm{x}^{\prime}$, temperatura $36,8^{\circ} \mathrm{C}$ axilar, saturación de oxigeno $98 \%$

Medidas antropométricas: peso de 79 $\mathrm{kg}$, talla $1.60 \mathrm{~cm}$

Biotipo: endomórfico

Índice de masa corporal: 30,86, obesidad I

Uñas rosadas cortas con llenado de 3 seg

Vello distribución androide en tórax, muslo y barbilla

Piel: elástica, disminuida con grosor, aumentado de acantosis nigricans

Diámetro de la cabeza antero posterior aumentado

Neurológico valorar el par II

Visión de túnel por la hemianopsia

Tono hipotonía, debilidad muscular

Marcha negativa

Laboratorio: hormona de crecimiento IGF1 1194(25-39), 114-492 
Crecimiento 14.7-0.0

Biometría

Cuantificación de la hormona hipofisiaria, tiroidea

Rayos x

Resonancia magnética

Prolactina 89-1.90-25

Macroadenoma hipofisiario

+acromegalia

\section{Anexo E. Caso clínico 5: abdomen}

Paciente femenina de 45 años sin antecedentes de importancia, ingresa a urgencias por presentar dolor de aparición insidiosa en hipocondrio derecho, moderado y opresivo, irradiado a flanco y a la región lumbar con movimiento exacerbado, abdomen globoso, blando, depresible, palpación profunda, timpanismo generalizado, paraclínicos dentro de los valores normales.

$\underline{\text { Antecedentes familiares }}$

Hepatopatías

$\underline{\text { Examen físico }}$

Tensión arterial: 150/80mmHg
Edema parpebral, obesidad generalizada

Dolor abdominal

Fiebre de $39^{\circ} \mathrm{C}$, cansancio, vómito, dificultad para respirar

Disminución en la cantidad de orina

Meteorismo (ruidos intestinales aumentados)

Sonidos son resonantes

\section{Antecedentes psicosociales}

Consume alcohol desde los 20 años, múltiples parejas sexuales, más de seis al mismo tiempo.

Con presencia de un abultamiento paralelo al ligamento inguinal.

Abdomen globoso

Edema

Disminución de la orina

Realizar prueba de onda ascítica

Ascitis + por proteinemia 